\title{
Transmissive optical fiber magnetic field sensor based on ferrofluids
}

\author{
Anna Dudus, Robert Blue, Deepak Uttamchandani \\ Centre for Microsystems and Photonics \\ Department of Electronic and Electrical Engineering, University of Strathclyde \\ Glasgow, G1 1XW, Scotland, UK \\ Corresponding author: d.uttamchandani@strath.ac.uk
}

\begin{abstract}
A compact optical fiber magnetic field sensor is reported which relies on the magnetic field induced displacement of a ferrofluid lying in the gap between two single mode optical fibers (SMFs) that are aligned face to face. The ferrofluid displacement alters the coupling of light from the input optical fiber to the output optical fiber. When the applied magnetic field changes from $0 \mathrm{mT}$ to $10 \mathrm{mT}$ the optical attenuation changes from $0 \mathrm{~dB}$ to $-28 \mathrm{~dB}$.
\end{abstract} sensor

Keywords-ferrofluid, magnetic field sensor, optical fiber

\section{INTRODUCTION}

In recent years there has been increasing interest in developing optical fiber magnetic field sensors by combining ferrofluids [1] with different types of optical fibers. Ferrofluids consist of a stable colloidal suspension of surfactant-coated ferromagnetic particles of dimensions around $10 \mathrm{~nm}$ suspended in a liquid medium which either can be water based or oil based. The ferrofluid has no remanence allowing the development of fully reversible and accurate sensing systems. Optically, the high concentration of magnetic nanoparticles in the fluid renders the fluid opaque at visible and near-infra-red optical wavelengths. However they can be diluted to increase optical transparency.

Application of a magnetic field results in the alignment of the randomly orientated magnetic moments, leading to a change in refractive index of the ferrofluid. This magnetooptic effect has been used in conjunction with fiber optic technology to realize an assortment of magnetic field sensors. For instance, fiber sensors have been reported based on submerging a microfiber knot into a ferrofluid [2], dipping single-mode fiber (SMF) tapers into a ferrofluid, $[3,4]$, using a ferrofluid as an outer cladding of an etched multimode (MM) fiber, a coreless multimode fiber in a $\mathrm{SMF} / \mathrm{MMF} / \mathrm{SMF}$ optical structure $[5,6]$ or a fiber grating etched within a D-shaped fiber [7]. These sensors require complex modification of the basic commercial fiber optic structure. In this paper we report a fiber optic sensor which does not use the magneto-optic property of ferrofluids, like the above sensors, but has a different operating principle, namely the physical motion of the fluid as a whole upon the application of a magnetic field. This operating principle has been used before for magnetic field sensing [8] but with the ferrofluid infiltrated within a microstructured optical fiber, which again requires complicated microfabrication techniques. Our fiber gap device is a simpler format of potentially lower cost system.

The wide diversity of applications of magnetometers such as military, industrial, power generation, geological surveying, and biomedical applications [9] can benefit from the advantages of optical sensors, especially immunity to electromagnetic interference, multiplexing, remote sensing and corrosion resistance. Additionally, fluidics can offer the advantages of developing sensing devices having no mechanical moving parts and being amenable to miniaturisation.

\section{FIBER OPTIC SENSOR DESIGN}

An externally applied magnetic field will cause the randomly orientated magnetic moments of the ferrofluid to align making the fluid move towards the region of highest magnetic flux. We have used this property to realize an extrinsic fiber optic sensor design by using a ferrofluid to create a movable fluidic "shutter" between two single-mode fibers (SMFs). This optical shutter consists of a plug of diluted, but still opaque, ferrofluid placed in direct contact with a plug of transparent fluid (glycerin-water solution) with which it is immiscible. This diluted ferrofluid comprises a solution of CHE006 ferrofluid (Rapid Electronics Ltd) mixed with mineral oil in the ratio 1:30. This optimum dilution ratio was reached through repeated experimentation and the final dilution ratio was selected so the fluid does not leave a residue on the channel glass wall but at the same time being opaque enough to achieve high optical attenuation. The fluids were housed in a miniature rectangular glass capillary channel (VitroTubes ${ }^{\mathrm{TM}}$, VitroCom Inc. USA) with transparent walls. The glass capillary had the inside diameter of $1 \mathrm{~mm}$ and the thickness of glass walls are $0.2 \mathrm{~mm}$. Both fluids also have the same refractive index, around 1.450 (measured with an Abbe refractometer), which is achieved by careful dilution of the ferrofluid and of the glycerin. By matching the refractive indices of the two fluids, unwanted interferometric effects, 
which can generate interferometric noise in the sensor, are reduced.

This liquid shutter system was translated (actuated) in the fluidic channel by a third liquid plug which comprised of an undiluted (100\%) oil-based ferrofluid (CHE006), as shown in Fig. 1. This undiluted ferrofluid plug was also immiscible with the glycerin-water solution with which it makes contact, and this plug moved under the influence of an applied magnetic field.

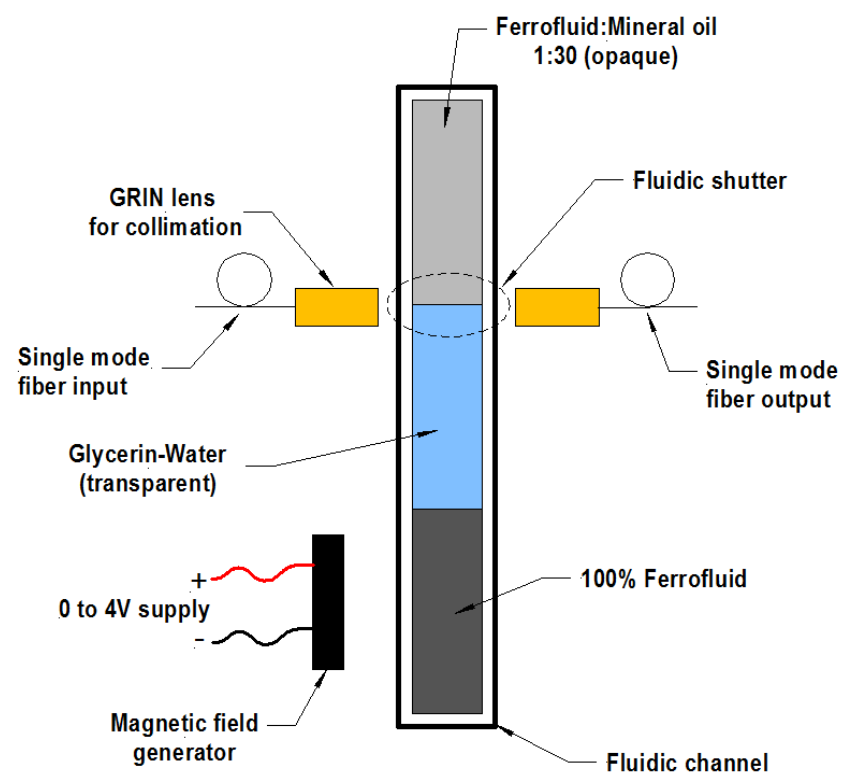

Fig. 1 Ferrofluid-based fiber optic magnetic field sensor layout

This overall fluidic assembly was positioned in the gap between two graded-index (GRIN) lensed SMFs aligned on opposite sides of the fluidic channel. The sensor was designed to operate as follows: the magnetic field being measured displaced the undiluted ferrofluid plug which pushed the glycerin/diluted ferrofluid shutter and when opaque shutter increasingly covers more of the light path, less light reaches the receiver lens. By measuring the light transmittance between the fibers with varying magnetic field, a fiber optic magnetic field sensor is realized.

Fig. 2 depicts a collimated light distribution in this shutter system which can be presented via a Gaussian function that adopts the form:

$$
A\left(x_{1}\right)=\int_{3 \sigma}^{x_{1}} \frac{1}{\sigma \sqrt{2 \pi}} \exp \left(-\frac{(x-\mu)^{2}}{2 \sigma^{2}}\right)
$$

where $A\left(x_{1}\right)$ is the attenuation when the opaque shutter blocks the light from position $3 \sigma$ to position $x=x_{1}$. Taking the diameter of the GRIN lens face to be $6 \sigma$ and equal to 1.2 $\mathrm{mm}$ yields a value of $3 \sigma=0.6 \mathrm{~mm}$ while $x_{1} \in(-3 \sigma, 3 \sigma)$. The theory predicts that when opaque shutter increasingly covers more of the light path, less light reaches the receiver lens but according to the Gaussian function never reaches 0 $\%$. Therefore when shutter translates $1.2 \mathrm{~mm}$, which is the diameter of the light beam, the transmission decreases only to $0.27 \%$.

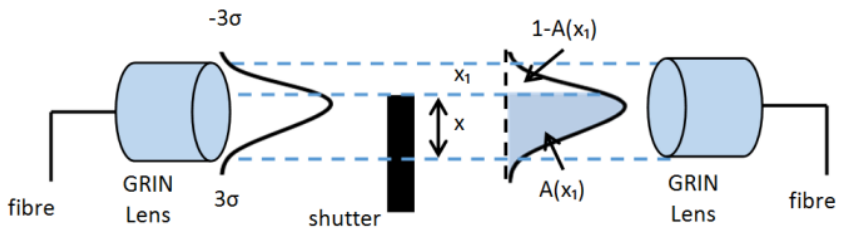

Fig. 2 Model of the Gaussian light distribution in a shutter system

\section{EXPERIMENTAL PROCESS}

The experimental process used an optical light source connected to the fiber optic magnetic field sensor, and an optical detector whose output was connected to a datalogger and computer. The optical source used was an erbium doped fibre amplifier (EDFA). An EDFA - which is a broadband optical source (1525 to $1560 \mathrm{~nm})$ - was employed for use with the single-mode-fibers and in order to reduce the effect of stray reflections interfering with each other to produce interferometric noise. Such noise is significantly reduced when a broadband optical source is used compared to when a narrow wavelength laser is used as the optical source. The light from the EDFA was launched into one of the GRIN lensed single mode fibers which collimated the optical beam as it traversed through the ferrofluid based sensor. On the other side of the sensor the second GRIN lensed fiber collected the transmitted light and guided it to a photodetector/amplifier (LNP-2, Optosci Ltd, UK). The output of the photodetector was recorded over time by a PicoScope data-logger (Pico Technology Ltd, UK) linked to a computer as shown in Fig. 3. The magnetic field strength of a electromagnet was measured and profiled with a Gaussmeter (GM07/GM08, Hirst Magnetic Instruments) and the magnet orientated to give a maximum displacement of the ferrofluid. The electromagnet was then offset $3 \mathrm{~mm}$ laterally from the fluidic channel and the magnetic field was varied linearly over the range $0 \mathrm{mT}$ up to $10 \mathrm{mT}$ by incremental step changes in the applied voltage to the electromagnet.

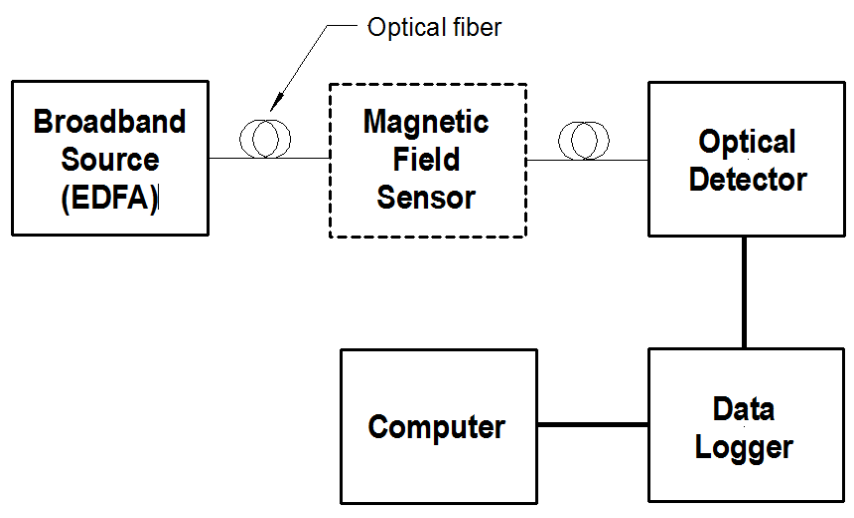

Fig. 3 Schematic of experimental setup 


\section{RESULTS AND DISCUSSION}

The experimental results are shown in Fig 4 where optical attenuation is plotted against the magnetic field strength in $\mathrm{mT}$. This is a relative measurement of optical transmission through the system. For zero applied magnetic field, the power received at the optical detector was recorded as a baseline measurement. As the magnetic field was increased in steps, the light received by the photodetector reduced and for each value of magnetic field, the light power at the photodetector was again recorded. The received light power was then converted into an optical attenuation value with reference to the light power measured at zero magnetic field. In this way, the overall trend of the sensor could be recorded without using reference to specific values of optical power.

As can be seen from Fig 4, for a magnetic field close to 10 $\mathrm{mT}$ the optical attenuation reaches up to $28 \mathrm{~dB}$. On the right hand vertical axis of Fig 3, the displacement of the ferrofluid has also been measured and recorded giving a useful indication of how the magnetic field strength translates to a physical displacement of the ferrofluid "shutter". The optical sensor attenuation response is non-linear for the linear displacement of the ferrofluid by the increasing applied magnetic field. This arises due to the Gaussian nature of the optical beam.

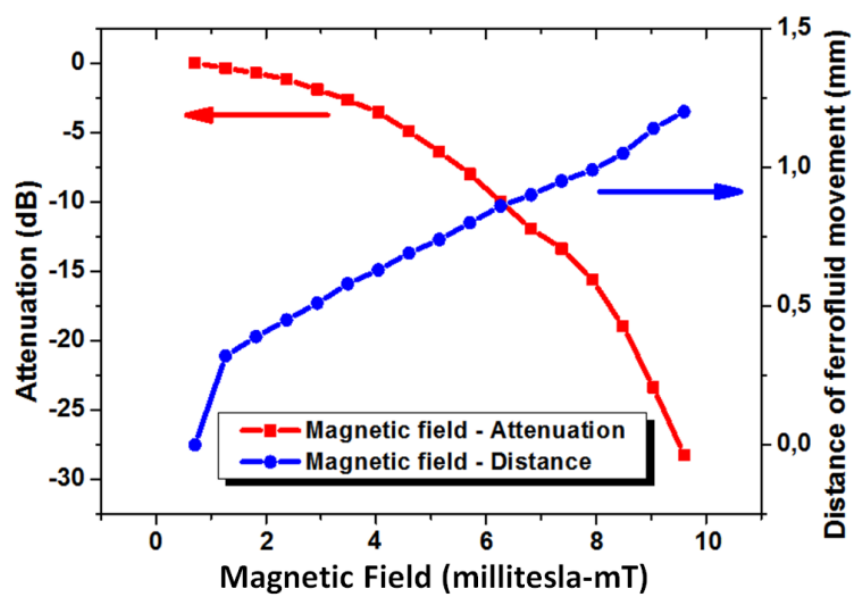

Fig. 4 Fiber optic magnetic field sensor response to changing external magnetic field strength

Further experimentation revealed that pre-treating the fluidic channel with a hydrophobic layer (Aquapel) the three liquid mediums can be reduced to two by switching to an undiluted water-based ferrofluid (EMG 605, Ferrotec Corp) as the opaque shutter and the second medium a mineral oil (Sigma Aldrich M3516) working as the transparent side of the shutter. The refractive indices of these media were found to be similar which reduced interferometric noise and an attenuation of $25 \mathrm{~dB}$ for an applied magnetic field of $10 \mathrm{mT}$ was experimentally measured for this prototype device.

\section{CONCLUSION}

We have demonstrated the concept of a ferrofluid based fiber optic magnetic field sensor which, in contrast to other similar devices, does not base its operation on magnetooptical effects i.e. change in refractive index with magnetic field. Our design is based on the physical movement of a ferrofluid "shutter" which is made to move when part of the fluidic shutter arrangement is exposed to a magnetic field. The movement of the shutter changes the light power transmitting across the sensor. This sensor is therefore based on light intensity measurements only and is therefore not exposed to interferometric noise which has to be solved in optical phase based fiber sensors. Our sensor work operates from 0 to $10 \mathrm{mT}$ and at $10 \mathrm{mT}$ magnetic field the optical attenuation is $28 \mathrm{~dB}$. This sensor could be further miniaturized and addressed by broadband LED and low cost detector and offer a simpler, less expensive format for an optical magnetic field sensor.

\section{REFERENCES}

[1] C. Scherer and A. M. Figueiredo Neto, "Ferrofluids: properties and applications," Braz. J. Phys. 35 (3A), 718727 (2005).

[2] X. Li and H. Ding, "All-fiber magnetic-field sensor based on microfiber knot resonator and magnetic fluid," Opt. Lett. 37 (24), 5187-5189 (2012).

[3]. A. Layeghi, H. Latifi, and O. Frazão, "Magnetic field sensor based on nonadiabatic tapered optical fiber with magnetic fluid," IEEE Photonics Technol. Lett. 26 (19), 1904-1907 (2014).

[4] M. Deng, D. H. Liu, and D. C. Li, "Magnetic field sensor based on asymmetric optical fiber taper and magnetic fluid," Sens. Actuators A Phys. 211, 55-59 (2014).

[5] H. Wang, S. Pu, N. Wang, S. Dong, and J. Huang, "Magnetic field sensing based on singlemode-multimodesinglemode fiber structures using magnetic fluids as cladding," Opt. Lett. 38 (19), 3765-3768 (2013).

[6]. Y. Chen, Q. Han, T. Liu, X. Lan, and H. Xiao, “Optical fiber magnetic field sensor based on single-modemultimode-single-mode structure and magnetic fluid," Opt. Lett. 38 (20), 3999-4001 (2013).

[7] L. Gao et al., "Long-period fiber grating within Dshaped fiber using magnetic fluid for magnetic-field detection," IEEE Photonics J. 4 (6), 2095-2104 (2012).

[8] A. Candiani, M. Konstantaki, W. Margulis, S. Pissadakis, "An optofluidic magnetometer developed in a microstructured optical fibre," Opt. Lett. 37 (21), 4467-4469 (2012).

[9] J. Lenz and A. Edelstein, "Magnetic sensors and their applications," IEEE Sens. J. 6 (3), 631-649 (2006). 